\title{
Aktuelle Therapiemöglichkeiten
}

Die Behandlungsmöglichkeiten der Parkinson-Erkrankung werden zunehmend umfassender und komplexer. Über den Stellenwert der Therapiemöglichkeiten bei den unterschiedlichen Stadien des Parkinson-Syndroms und das Auftreten „nicht motorischer“ Symptome bei Morbus Parkinson führten wir ein Gespräch mit Prim. Dr. Dieter Volc, Leiter des Parkinson-Zentrums an der Privatklinik Confraternität in Wien.

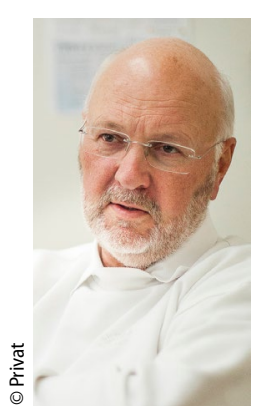

Prim. Dr. Dieter Volc
Welche Krankheitsstadien werden bei M. Parkinson unterschieden?

Volc: Für die Betroffenen ist wichtig zu wissen, dass anfangs eine einseitige langsam fortschreitende Symptomatik besteht, die sehr gut behandelbar ist. Mit der Zeit kommt auch die zweite Seite hinzu. Nach jahrelangem Verlauf kann es dann zu motorischen Komplikationen kommen, die eine Änderung des Behandlungsplanes nach sich zieht. Schwieriger wird es in späten Phasen, weil dann auch ein kompliziertes Behandlungsschema nicht immer zur Symptomfreiheit führt. Der Krankheitsverlauf ist ein schleichender, es gibt keine Schübe und keine wirklichen Stadien, die bei jedem Menschen nach gewissen Zeiten auftreten. Für die klinische Einteilung des Schweregrades gibt es Skalen und Untersuchungsprotokolle, die sich an der Gesamtheit der Symptome orientiert. Im Stadienprotokoll von Hoehn \& Yahr unterscheidet man einseitige Symptome $(1,0)$, beidseitige Symptome $(2,0)$, schwerere $(3,0)$, schwere $(4,0)$ und sehr schwere $(5,0)$ Symptomatik. Bei 1 und 2 gibt es noch den Zusatz von Balancestörungen mit 1,5 und 2,5 .

Welche Therapiemöglichkeiten kommen in den frühen Krankheitsphasen zum Einsatz?

Volc: Grundsätzlich steht derzeit nur eine symptomatische Therapie zur Verfügung. Wir können also Beschwerden im Zaum halten, aber weder ist eine Verlaufsmodifikation derzeit nachweislich möglich, noch gar eine Heilung. Etwas abhängig vom biologischen Alter kommt bei Jüngeren zunächst ein Dopaminagonist zur symptomatischen Therapie in Frage, später kommt Levodopa dazu. Bei Älteren wird man gleich mit Levodopa beginnen. Zusätzlich stehen MAO-B - Hemmer, Amantadin und COMT-Hemmer als Zusatzstoffe für die Feinabstimmung zur Verfügung.Das frühzeitige Heranführen an Bewegung und Physiotherapie sind besonders wichtig, weil das für den Rest des Krankheitsverlaufes bedeutend ist.

Was ist bei den fortgeschrittenen Stadien in der Praxis besonders zu beachten und welche Behandlungsoptionen stehen zur Verfügung?

Volc: Levodopa ist auch 55 Jahre nach der ersten Anwendung weltweit immer noch der Goldstandard der Parkinsonbehandlung und ohne geht es praktisch nie. Durch die verminderte Speicherfähigkeit der Neuronen für Dopamin sind Fluktuationen und Dyskinesien dann ein häufiges Problem und die Therapieschemata werden schwieriger mit kurzen Einnahmeintervallen und schlechterer Steuerbarkeit. In diesen Fällen muss man auch über die invasiven Therapiemethoden nachdenken.

Viele Betroffene empfinden die „nicht motorischen" Symptome als belastend. Es wird geschätzt, dass $40 \%$ der Patienten unter Depressionen leiden. Benötigen die Betroffenen neben der Parkinson-Medikation eine zusätzliche gezielte Behandlung?
Volc: Die nicht motorischen Beschwerden sind in der Tat meist belastender. Verstärkter Speichelfluss, Schmerzen, Blasenstörungen, Obstipation und vor allem Schlafstörungen quälen ganz besonders und sprechen auf dopaminerge Medikation meist auch gar nicht an. Das ist aber nicht unbedingt der Grund für Depressionen. Die kommen grundsätzlich vor, können durch schlechte Krankheitsverarbeitung verstärkt sein, sie können aber auch durch niedrige Dopaminspiegel ausgelöst werden. Hier ist es wichtig, dass auf Parkinson spezialisierte ärztliche Beratung beansprucht wird, da Therapieansätze einerseits tatsächlich Antidepressiva sein können, andererseits aber auch eine modifizierte DOPA-Behandlung.

Wie würden Sie Ihr Therapieziel bei M. Parkinson definieren?

Volc: Mein Ziel ist immer ein möglichst beschwerdefreies Leben mit guter Qualität und möglichst wenig Medikamenten zu ermöglichen. Das bedingt vor allem eine gute Zusammenarbeit der Betroffenen, deren Angehörigen und Betreuungspersonen, einer fachkundigen ärztlichen Beratung unter Einbeziehen aller Beteiligten in Kombination mit einer vernünftigen Kombination mit Physiotherapie, Ergotherapie, psychologischer Betreuung und Selbsthilfeangeboten.

psychopraxis. neuropraxis 2016 · 19:146 DOI 10.1007/s00739-016-0342-x

Online publiziert: 10. August 2016 C Springer-Verlag Wien 2016 